\title{
Monitoring the first dockless bike sharing system in Greece: Understanding user perceptions, usage patterns and adoption barriers
}

\author{
Efthimios Bakogiannis ${ }^{1}$, Maria Siti ${ }^{1}$, Stefanos Tsigdinos ${ }^{1}$, Avgi Vassi ${ }^{1}$, \\ Alexandros Nikitas ${ }^{2 *}$, \\ ${ }^{1}$ National Technical University of Athens \\ E-mail: ebako@mail.ntua.gr \\ E-mail: sitim.atm@gmail.com \\ E-mail: stef.tsigdinos@ gmail.com \\ E-mail: avgi.vassi@gmail.com \\ ${ }^{2}$ University of Huddersfield \\ E-mail: A.Nikitas@hud.ac.uk (corresponding author)
}

\begin{abstract}
The city of Rethymno in Crete is the first Greek city to host a Dockless Bike Sharing System (DBSS). This is a scheme that means to promote sustainable mobility and help mitigating high levels of car traffic induced particularly during summer by a consistently increasing number of tourists. The present paper examines the efficiency of this DBSS aiming at: a) analysing the current usage patterns, $b$ ) identifying and discussing the various reasons that encourage or deter its usage and c) capturing a few key user perceptions reflecting and affecting scheme acceptance and usage. The study adopts a mix-method approach consisting of secondary data analysis, field observations and a quantitative survey, completed by 534 DBSS users. The findings illustrate that DBSS is used primarily for short-distance trips (up to $1,5 \mathrm{~km}$ ) and very short rentals (up to $15 \mathrm{mins}$ ). Traffic safety concerns and limitations in the existing cycling infrastructure are two of the factors adversely affecting the scheme's usage, since many questioned whether the scheme was an effective investment under the present situation with the given constrains of Rethymno's transport system. The majority of both frequent and occasional users thought that the scheme is affordable, easy to use and suitable for both tourist and local populations. These findings constitute the first ever footprint of DBSS usage in Greece and can be used as an input for delivering appropriate policy interventions in future urban transportation strategies looking to promote and reinforce bike sharing usage and increase cycling uptake. The paper also offers valuable guidance to mobility providers about how bike sharing businesses can prosper long-term in an environment where shared mobility schemes constitute novel socio-technical interventions.
\end{abstract}

Keywords: Dockless bike sharing, bike sharing, public bicycles, sustainable transport, shared mobility

\section{Introduction}

The typical modern city suffers from efficiency and sustainability problems (Jabareen, 2006). One of the key reasons behind this challenging reality is that for decades now urban transportation has been strongly dependent on motor vehicles. An automobile-oriented planning approach made cities "captive" to private cars (Marshall, 2005) and redefined urban landscapes establishing the concept of the 'automobile city' where cities began to decentralise and disperse (Newman and Kenworthy, 1996). This car-dictated mobility paradigm has led to an unattractive street environment, urban sprawl, inadequate public transport service, intense traffic congestion, road accidents and mobility inequalities (Brueckner, 2000; Nieuwenhuijsen and Khreis, 2016). A new mobility ethos should be established therefore, for cities looking to effectively address these challenges, which among others will require from policy-makers and mobility providers to promote voluntary travel behaviour change via powerful active and shared transport initiatives (Nikitas et al., 2019). 
This new ethos reflects the rise of a powerful innovative planning perspective concerning cities and their future; this is the concept of sustainable mobility (Bannister, 2008). Sustainable mobility is based on embracing alternative means of transport along with limiting car use. Its purpose is to safeguard environmental perseverance, social equity and economic efficiency (Gudmundsson, 2004) and contribute positively to communities and their built environments (Attard \& Shiftan, 2015).

A crucial pillar of sustainable mobility, which constitutes an independent and environmentally friendly way of travelling around the city, is cycling (Bakogiannis et al., 2017; Gossling \& Choi, 2015; Shokoohi \& Nikitas, 2017; Vlastos \& Milakis, 2006). In order to promote this transport mode, numerous cities worldwide have installed bike sharing systems (BSS) anticipating several benefits for their residents and their visitors (Parkes et al., 2013). These refer to flexible mobility, congestion reduction, physical activity increase among others (Craig et al., 2017; Shaheen et al., 2010). According to Pucher et al. (2010), bicycling has increased in cities that have implemented bike sharing programmes in parallel with improvements in bicycling facilities.

A new type of BSS that has emerged over the last four years, which adds, in theory at least, an unprecedented door-to-door dimension in public bicycle programmes, is the dockless bike sharing system (DBSS) or free-floating bike sharing (FBSS) system (Acquier et al., 2017). This is an innovative bike sharing form that does not require docking stations, ensuring even more flexibility, and therefore contributing to the solution of the 'last mile' problem in urban transportation (Pal and Zhang, 2017). However, when compared with dockbased systems, DBSS has significantly more theft, misuse and vandalism-related problems (Duran-Rodas et al., 2018; Nikitas, 2019).

BSS has been typically framed and studied as a solution suitable for larger metropolitan areas but rarely tested for the context of smaller and medium cities with a few notable exceptions (e.g. Claufield et al., 2017; Nikitas, 2018; Nikitas et al., 2016). Also because of its very recent emergence DBSS literature is still rather scarce (Li et al., 2018). This work means to address these two critical research gaps in a climate where the concept of DBSS is facing some serious criticism lately about its genuine capacity to be a viable long-term solution (e.g. de Chardon, 2019) but may still be an effective shared use mobility apparatus if approached, designed, implemented and managed in the right way (Nikitas, 2019).

The tourist city of Rethymno, a medium-sized Greek city in the island of Crete, has recently established a DBSS in its city centre, in order to promote cycling and sustainable mobility in general. This is the first Greek city to implement such a pioneering solution with the participation of the private sector which is responsible for implementing and managing its operation. The present paper investigates the efficiency and the attractiveness of this established system aiming at: a) depicting the current situation regarding its use, b) examining the various reasons that encourage or deter it, as well as c) capturing the users' perceptions about it. The research is based on travel data recorded by the scheme operator, field research and a quantitative survey answered by scheme users.

The paper consists of seven more sections. The next section reviews the emerging bike sharing literature, followed by a section that introduces the study location and the scheme specifics. The fourth section presents the mixed-method methodology employed. The fifth section presents the results of our analysis, the sixth section a discussion including recommendations for the key stakeholders and the seventh section acknowledges the study's strengths and weaknesses and our vision for future studies. The work ends with a conclusions section. 


\section{Dockless Bike Sharing Systems: A Brief Review}

Sharing economy is a concept spreading all over the globe; several platforms such as Airbnb, Couchsurfing, Uber, Lyft, to name a few are gaining recognition and attracting an increasing number of users (Hamari et al. 2016; Wang \& Zhang, 2012). In this context, BSS could be considered as the contribution of cycling in the sharing economy rationale. At the same time, BSS has morphed over the last decade at least to a critical component of current urban mobility management strategies (Shaheen et al., 2010) emerging as an important innovation reflecting and affecting urban public transport (Morton, 2018).

One of the main objectives of a BSS, underpinning this study, is to provide free or low-cost access to public bicycles for short distance trips in cities, removing the burden of costs and responsibilities relating to bike ownership. It is widely regarded as an alternative option to private car use that helps reducing traffic congestion, air pollution and noise levels (Shaheen et al., 2013; Vogel, et al., 2011; Wang and Zhou, 2017) ${ }^{1}$. Furthermore, bike sharing is linked to public transport either as an alternative transport mode or as a 'last mile' solution, connecting commuters to public transport hubs (Liu et al., 2012; Ma et al., 2019). Bike sharing has boomed over the last decade, experiencing the rapidest growth of any mode of transport in history (Bakogiannis et al., 2016; Midgley, 2011). According to DeMaio (2009) the introduction of BSS has profound impacts on "creating a larger cycling population, increasing transit use, decreasing greenhouse gases, and improving public health". Bike sharing constitutes both an alternative transport option and a means to increase cycling by integrating bike use better within the whole transportation system (Shaheen et al., 2012). This considerable flexibility has led to a great increase of its popularity.

In this context, a BSS, can be defined as "a scheme referring to the provision of affordable short-term access to locally branded bicycles on an 'as-needed' basis that could extend the reach of public transit services to final destinations and be a door-opener for increased bicycle usage" (Nikitas, 2018). Moreover, BSS can be classified according to financing models followed (public, private, or public-private partnership), ownership, operator and operational model, scale and range (Ricci, 2015).

BSS can bring about several benefits for the urban environment and its residents or visitors (Shaheen, et al., 2010). Some of them are the following: time savings and convenience for travelers (Fishman et al., 2013), mitigation of traffic congestion (Hamilton \& Wichman, 2018), promotion a healthier lifestyle (Midgley, 2011), reduction air and noise pollution (Shaheen et al., 2013), increase of traffic safety level (Fishman \& Schepers, 2014). Regarding users' frequency, it should be mentioned that BSS subscribers may view bike sharing as an occasional adjunct to their primary and secondary transport modes (Fishman, 2016; Nikitas et al., 2016). The most important factors affecting bike sharing usage for some researchers are weatherrelated variables, land-use and built environment characteristics (Wang et al., 2018). Bike sharing systems should also be carefully developed to appreciate the quality and timely interplay between the physical design of the system and the provision of services being offered (Zhang et al., 2015).

BSS are changing radically through the years. Some researchers have categorised the evolution of BSS into four generations (Parkes et al., 2013). The first generation of BSS was "The White Bike program" in Amsterdam, which was characterised by no payment or security functions (Davis, 2014). Second-generation programmes adopted a coin deposit system (similar to trolleys at a supermarket). The first large-scale second-generation programme launched in Copenhagen in 1995, but the anonymity exposed the system to theft (DeMaio, 2009).

\footnotetext{
${ }^{1}$ However, there is research (e.g. Fishman et al, 2014) suggesting that BSS can also, in some cases, increase traffic congestion due to fleet rebalancing needs that are typically achieved through the use of trucks and trailers.
} 
The challenges faced by these first two generations resulted in the development of the thirdgeneration systems, which are characterised by dedicated docking stations, as well as automated credit card payment and other technologies to allow the tracking of each bicycle (Shaheen, Cohen \& Martin, 2013). It is considered that these elements, in conjunction with growing public policy interest in cycling (Pucher \& Buehler, 2012), have enabled the fast growth of BSS across the globe (Shaheen \& Guzman, 2011). The fourth generation is recently introduced, including dockless systems, easier installation, power assistance and transit smartcard integration (Parkes et al., 2013).

When focusing on DBSS it should be noted that these particular systems are considerably different from the traditional schemes in many aspects. For instance, in terms of operation, dockless bicycles possess the following characteristics; they integrate mobile payment and global positioning system (GPS) tracking into the system, thus increasing the ease of use and management (Shen, et al., 2018). The mobile applications allow users to unlock and lock these GPS-enabled share bikes anywhere in the city when their rides begin or end (Pal and Zhang, 2017; Reiss et al., 2015). Furthermore, in order to accommodate customers, a credit system can also be established (Shen, et al., 2018).

The unique characteristics of DBSS present several benefits, fixing some drawbacks of the traditional system. Firstly, the potential of these bicycles to be parked anywhere means that the fleet size is not limited by the capacity of each docking station, thus providing greater chances of users to find available bicycles. It also provides more flexibility and potential for door-todoor services. This 'freedom' increases significantly users' convenience (Shi, et al., 2018). Moreover, the surpassing of these constrains can lead to using bike sharing for commuting and not only for leisure purposes (Fishman, et al., 2014). Finally, due to the high potential supply of bikes, a DBSS has a lower cost per bike than dock-based systems (Mooney, et al., 2019), therefore permitting better pricing policies to be implemented something that enhances the attractiveness of bike sharing and increases the affordability of transport as a whole. At the same time nonetheless, many DBSS systems, face more and significantly bigger problems of theft, misuse, vandalism than dockbased schemes, that led to many recent scheme closures and a full-scale retreat of mega-startup companies like the Chinese Ofo and Mobike from Europe (Nikitas, 2019). So, it should be noted that DBSS are not always superior in practical terms than dockbased systems; actually the latter seem as of now the more 'durable' brand of bicycles.

In the existing literature, there is an extensive amount of research about the traditional dockbased BSS (Li, et al., 2019), while the sustainability performance of new-generation dockless schemes is not comprehensively examined as yet (Nikitas, 2019; Shi, et al., 2018). According to van Waes, et al. (2018) station-based business models are well institutionalised but harder to scale up, while the dockless model has the greatest scaling potential, if institutional adaptations and geo-fencing technologies are successfully implemented. Furthermore, in dockbased schemes satisfaction is seriously affected by a set of factors like the mechanics of the bikes, the picking and dropping system, and the apps used to organise the service (Manzi and Saiben, 2018). At the same time dockless shared mobility models are potentially useful in generating participation, but face substantial technical, analytical, and communication barriers (Taleqani, et al., 2019) and higher vulnerability to mismanagement (Luo et al., 2019).

\section{Study Area}

\subsection{The City of Rethymno}

Rethymno is a cosmopolitan city in Crete, Greece, situated between the bigger cities of Heraklion and Chania that are hosting the two major international airports of the island. Rethymno is defined by strong networks of small to medium sized neighboring settlements, which influence daily mobility patterns. The Greater Rethymno's permanent population is 
55.525 inhabitants (ELSTAT, 2011), of which 32.468 live in the compact and densely built residential area of the city (Athanasopoulos \& Vlastos, 2015). Because of tourism activities, Rethymno is one of the most rapidly developing municipalities in the region; between 2001 and 2011 its population increased by $17 \%$. The significant influx of tourists during the summer months that in July 2012, accounted for 79,000 tourists (90\% of whom were foreigners), introduces new travel needs and a big increase in traffic. Due to a tradition of rather inefficient mobility management, this tourism-induced traffic leads to downgrading further the city's urban environment and calls for specific measures to address this seasonal transport problem. One of the steps for responding to this challenge is to add a non-motorised transport alternative that is inexpensive (i.e. small capital investment) and easy (in political and business terms) to introduce; thus Rethymno became the first city in Greece to implement a DBSS, as a significant step towards creating a more sustainable mobility culture that adds to its cosmopolitan identity.

\subsection{Rethymno's Transport System and DBSS}

The city because of its relatively small size has no tram or metro services to offer but hosts a wide range of taxi and bus services. Typical taxi costs are equivalent to $1.02 € / \mathrm{km}$ or in average $€ 4.5$ per trip. A single bus ticket for a local city ride costs $€ 0.7$. The city has launched in July 2019 a pilot electrical minibus (part of the EU project CIVITAS Destinations) and one of Greece's first electrical scooter-sharing schemes costing $€ 1$ per rental plus $0.15 € / \mathrm{min}$ of use. Car rentals are also popular especially for tourists. Rethymno is the only city in the region having an urban cycling network of $8.16 \mathrm{~km}$ routes in its key arteries and thus is the most procycling city in Crete in terms of its existing bicycling infrastructure although this is limited when compared with other European tourist destinations.

Rethymno's DBSS, Bikeazy, was introduced during the summer of 2018 and is associated with the CIVITAS Destinations programme. The fleet consists of 300 bikes; there were no serious fluctuations in numbers during the data collection and analysis phases. This is the largest fleet in any Greek city (dockless or not). All the bikes of the fleet have an integrated electrical battery. The bikes can be locked or unlocked through a mobile app. The system includes a wide range of bicycling parking racks, bicycling securing locations across the city and geofencing as mechanisms designed to reduce misuse. The prices for using the scheme are summarised below.

Table 1: DBSS access prices

\begin{tabular}{|l|r|l|l|l|r|}
\hline \multicolumn{2}{|l|}{ Subscriber packages } & \multicolumn{3}{l|}{ Occasional user packages } & \multicolumn{2}{l|}{ Visitors packages } \\
\hline Monthly subscription & $€ 14.90$ & First 15 minutes & $€ 1.00$ & One-day pass & $€ 19.90$ \\
\hline Yearly subscription & $€ 89.00$ & Every other 15 minutes & $€ 1.50$ & Three-day pass & $€ 29.90$ \\
\hline Every 15 minutes & $€ 0.50$ & & & Five-day pass & $€ 39.90$ \\
\hline
\end{tabular}

The DBSS fleet is managed, maintained and charged by a private contractor specialised in bike sharing services in Greece named Brainbox Technology. The private operator has signed an agreement with Rethymno's municipality government that has a supervisory role.

\section{Research Methodology}

This study adopts a mixed-method approach due to its multidimensionality, validation and triangulation merits that add to the breadth and depth of a research (Johnson et al., 2007). After a literature review that allowed the identification of knowledge gaps and enable us to elaborate meaningful and timely research objectives as described in the introduction this work examines the usage and acceptance patterns of Rethymno's Bikeazy combining the findings from three methods namely: a secondary data analysis, a series of observations and a brief quantitative 
survey. The methodological framework reviewing the three methods used is diagrammatically presented in Figure 1.
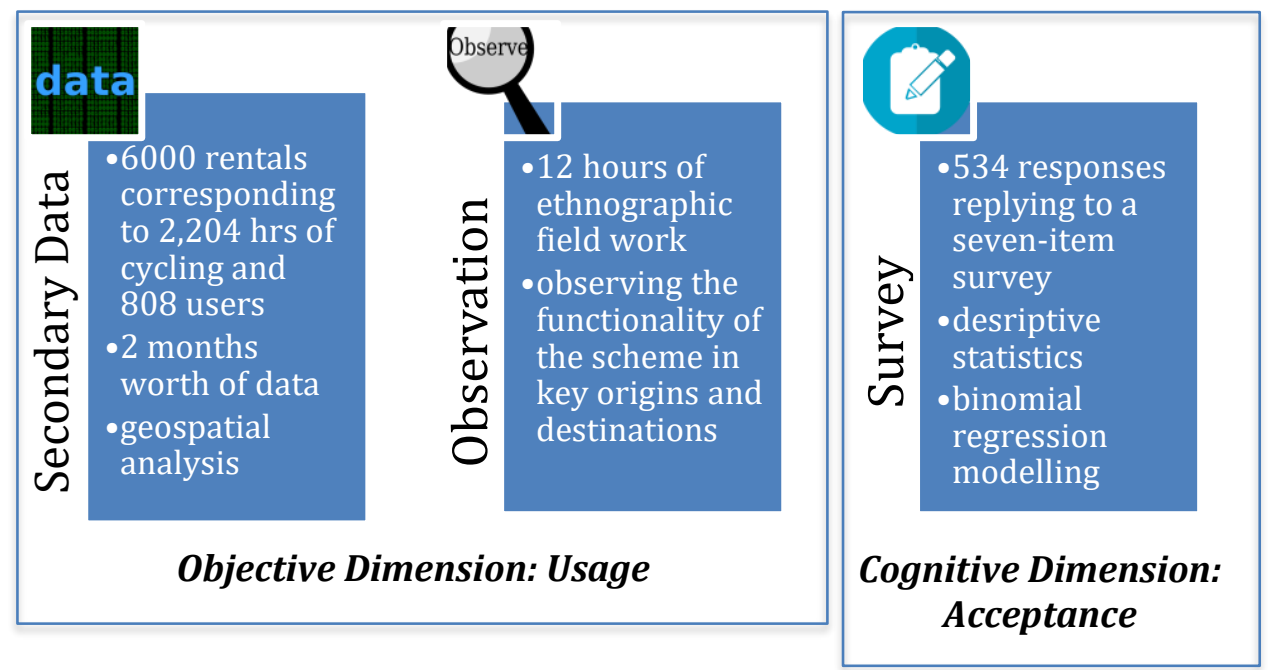

Figure 1: Methodological framework

The secondary data analysis refers to data regarding the actual use of dockless bicycles in Rethymno between the $30^{\text {th }}$ July and $30^{\text {th }}$ September 2018 . The usage data were provided directly by Brainbox Technology, the private contractor operating the scheme. The data refers to 6000 bicycle rentals made by the scheme's subscribers/users.

This analysis was supported and validated by a field research scheme of ethnographic nature. Around 12 hours of observations were conducted by the authors in teams of two to allow for a more objective recognition of the DBSS usage phenomena. This supporting, yet independent procedure, helped the research team to develop a real-life sense of how the DBSS system was performing in a regular basis and provides a qualitative dimension to the work. The authors visited many of the key, as defined by the secondary data, origins and destinations in the urban fabric of Rethymno to observe DBSS use and identify whether the Rethymno scheme was a well-functioning system operating without significant problems. The observations that took place in late September 2018 were primarily non-participatory and collected descriptive field notes recording user behaviour although all but one of the authors used the scheme themselves. The observational work mostly supports the secondary data findings (i.e. most service subscribers used the bikes for short trips) and recorded few incidents of misuse/vandalism and a general hesitation by most subscribers to use these bikes to their full pedal-assisting potential.

The two first methods of the study refer to usage and establish the objective dimension of the work looking primarily to usage patterns. This phase of the research offers a descriptive analysis of the general characteristics (bicycle fleet, system's users, and number of rents), spatial analysis of the geography of routes (traces, starting and ending points, density) and a presentation of the average trips' duration. The paper presents these findings in the form of diagrams and maps and evolves the understanding of the current usage patterns.

The second component of the work (i.e. the cognitive dimension) refers to a quantitative survey of seven questions administrated in January/February 2019 exclusively to DBSS users. This primary research phase, which examines user perceptions reflecting and affecting acceptance patterns, includes descriptive statistical and regression analyses of the survey data. The total sample consists of 534 fully completed responses and represents $66 \%$ of the system's users as 
recorded in $30^{\text {th }}$ September 2018 and over $25 \%$ of the subscriber population at the time of the survey collection making these results entirely representative of the user population. This extremely high recruitment/response rate was achieved because the survey launch was part of the municipality's event for promoting sustainable mobility in general and DBSS in particular; all the DBSS users were invited to this and were asked to fill in a survey. The event ${ }^{2}$ provided incentives (free day-passes) for people to subscribe in the scheme and then after using the bikes to fill in the survey. Also, all the major hotels with access to DBSS promoted the survey; hard copy surveys and collection boxes were available to them and the City Hall. The questionnaire sample was divided into two categories - frequent and occasional users - in order to obtain a more accurate account of the users' perceptions and understand how attitudinal differences relate to frequency of use.

\section{Analysis and Results}

\subsection{Analysis of the DBSS Usage: Secondary Data Analysis and Observations}

The first pillar of the analysis looks at the use of Bikeazy during its early days. The total number of registered DBSS users includes 808 residents and visitors of the city that had used one of the service packages of Bikeazy as presented in Table 1 . About $55 \%$ of these had access to an android operational system and the rest to iOS. In the examined two-month period 6,000 rentals were recorded.

The average rentals, per user equals to seven and per bicycle 20; both reasonable numbers for a brand-new system in a city with a non-established pro-cycling culture. The percentage of users with rentals above the average value is approximately $25 \%$, while the respective percentage concerning rents per bicycle equals to $40 \%$. Hence, it is rather clear that some users use the system more regularly than others and that many bicycles receive greater stresses (higher possibilities of severe damage) primarily due to being located more centrally. The maximum rents per user are 70 and the minimum just one, while the maximum rents per bicycle are 224 and the minimum is one. The number of rentals linked with the municipality's DBSS launch event that was promotional and offered free day-passes was 735 .

The average duration of a rental is approximately 22 minutes while the median duration was 6 minutes. Focusing further on the duration of trips, the trips can be categorised in seven timeperiod categories all of them referring to trips lasting up to 60 minutes; trips lasting above 60 minutes were very rare and were ultimately excluded from our analysis because some of them according to the operator may possibly be a result of a GPS error. The results of the trip duration evaluation are presented in Fig. 2.

\footnotetext{
${ }^{2}$ The event was part of the public engagement agenda that is critical for the European Commission-funded initiative SUMP (Sustainable Urban Mobility Plan) that helps cities to generate a strategy for better transport.
} 


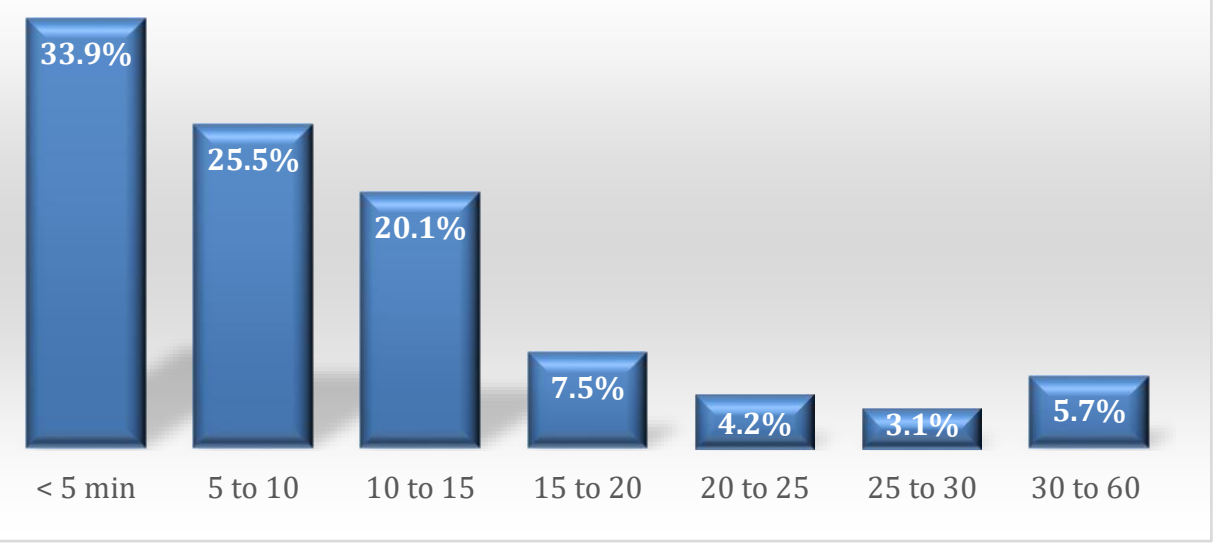

Figure 2: Categorisation of trips based on duration (in minutes)

The category with the highest percentage contains trips with duration up to 5 minutes $(33,86 \%)$. The next category includes trips lasting from 5 to 10 minutes $(25,47 \%)$. On the contrary, the category with the lowest percentage contains trips lasting between 25 to 30 minutes. Consequently, it seems that users prefer to choose DBSS for short distance trips and especially "last mile" trips. In the case of bigger distances, the users, choose DBSS as a complementary solution to other faster and "more convenient" means of transport (e.g. car or bus).

Regarding the spatial distribution of the trips according to their respective duration, a map showing origin points coloured depending on their duration was developed in Fig. 3.

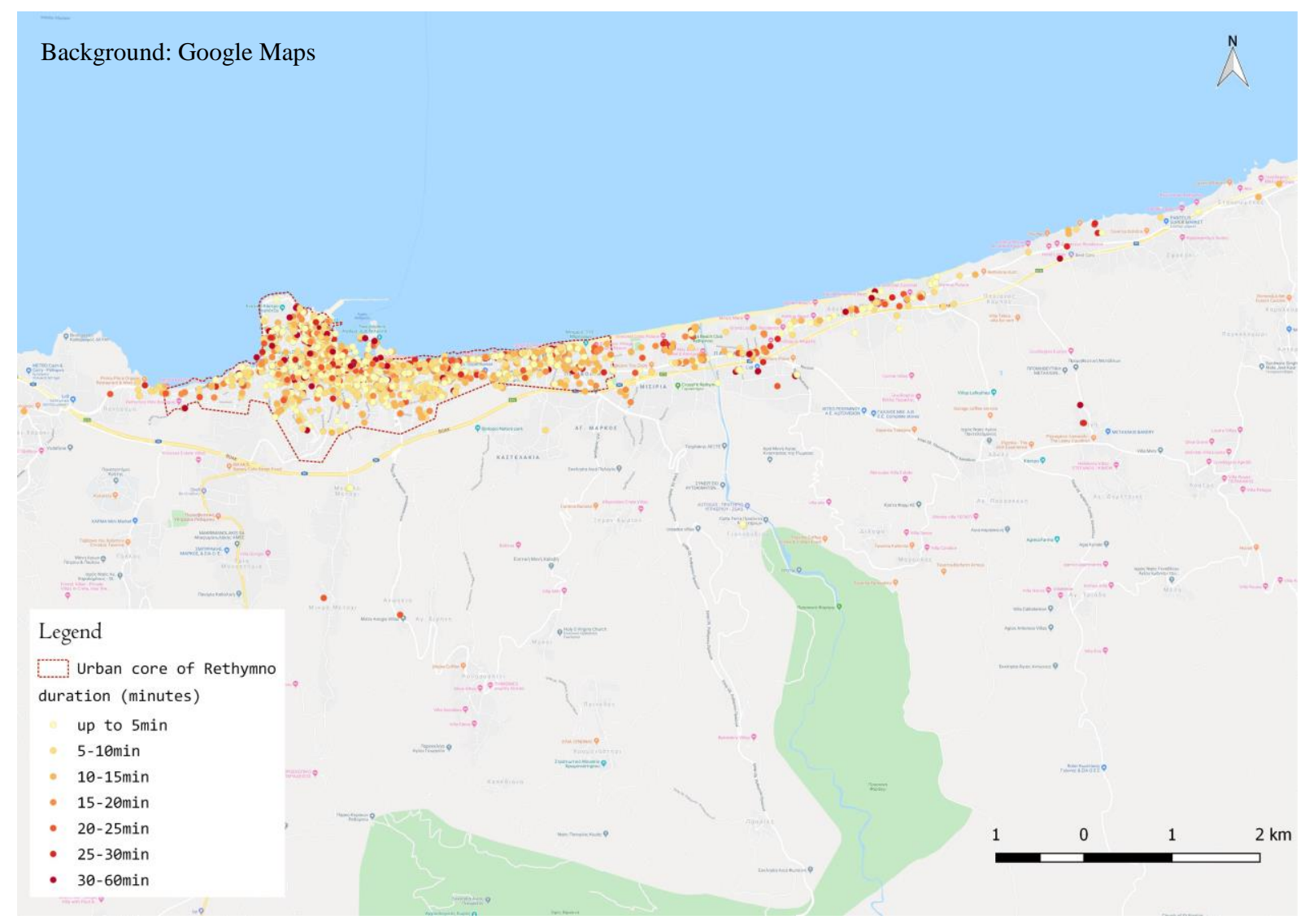

Figure 3: Origin points categorised by duration 
Longer trips are primarily located in the central area of Rethymno and especially in the Old Town, and in the east suburbs of the city (areas with denser tourism activities). On the contrary, shorter trips are dispersed in the overall city area. However, many of them are also located in the urban core of the city.

Regarding the routes recorded, the developed map (Fig. 4) depicts the traces of the $77 \%$ of the routes completed in the time period between $30^{\text {th }}$ July and $30^{\text {th }}$ September 2018. Results show that routes cover the whole city of Rethymno, including its west and east suburbs. Furthermore, routes spread to south areas as well. The horizontal range of routes (west to east) is $19.5 \mathrm{~km}$ and the vertical (north to south) is approximately $3.8 \mathrm{~km}$. Traces intensify in the main urban core and especially in the city center and the Old Town as well as along the seafront. Also, a significant density of traces is observed along the old national road (Regional Highway) which lays in parallel to the seafront. The total trip kilometers examined by the study equals to 7,759 $\mathrm{km}$. The maximum individual trip length equals to $28.3 \mathrm{~km}$, while the minimum is almost 0 kilometers. The average value of kilometers covered per trip is $1.7 \mathrm{~km}$. This fact, in coordination with the trip duration analysis, further supports the argument that the DBSS in Rethymno seems to be becoming a popular choice for short distance trips.

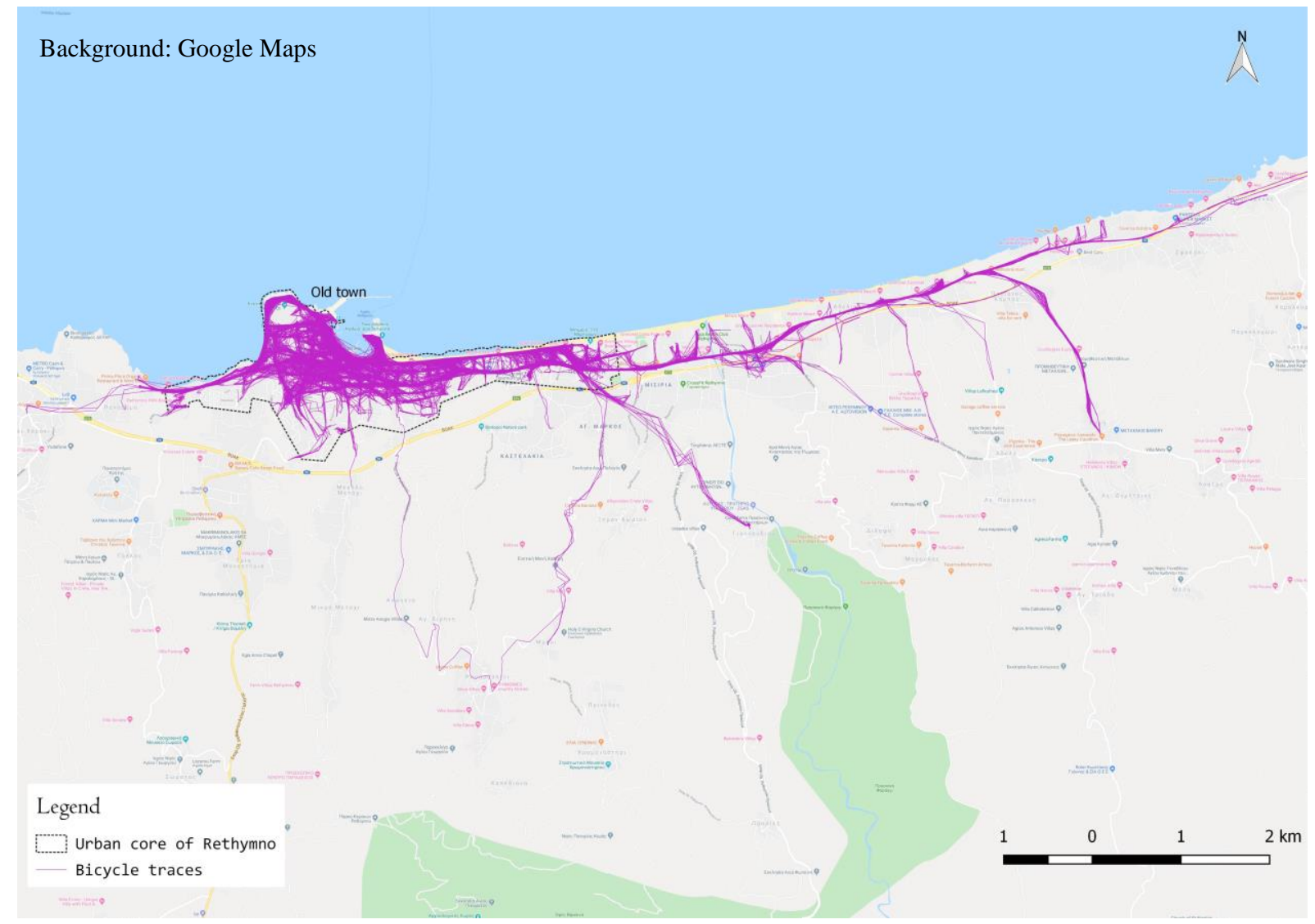

Figure 4: Traces of the DBS routes

The analysis includes the mapping of starting points' and terminal points' density, using the Kernel Density tool (heatmap) of QGIS for a 200 m radius. The Kernel density tool is an efficient way of identifying spatial clusters. The identification of these clusters can contribute to the comprehension of the supply and demand for bike sharing in the city. 
Figure 5 highlights the fact that both starting and ending points cover the whole area of the city, spreading towards all directions. This means that the spatial coverage of the system can adequately serve the urban and suburban area of Rethymno, facilitating the use of the system for a notable percentage of residents and visitors. Nevertheless, the use of bicycles does not take place similarly in the whole study area. On the contrary, two clusters arise in both cases (starting or terminal points). These clusters are both located within the urban core of the city; the first located in the central part of the city (Old Town and port) and the other in the eastern part of the urban core. The areas composing the clusters are mainly places with a high land-use mix, intense tourism activities, commercial and recreational activities as well as considerable historic interest (Old Town).

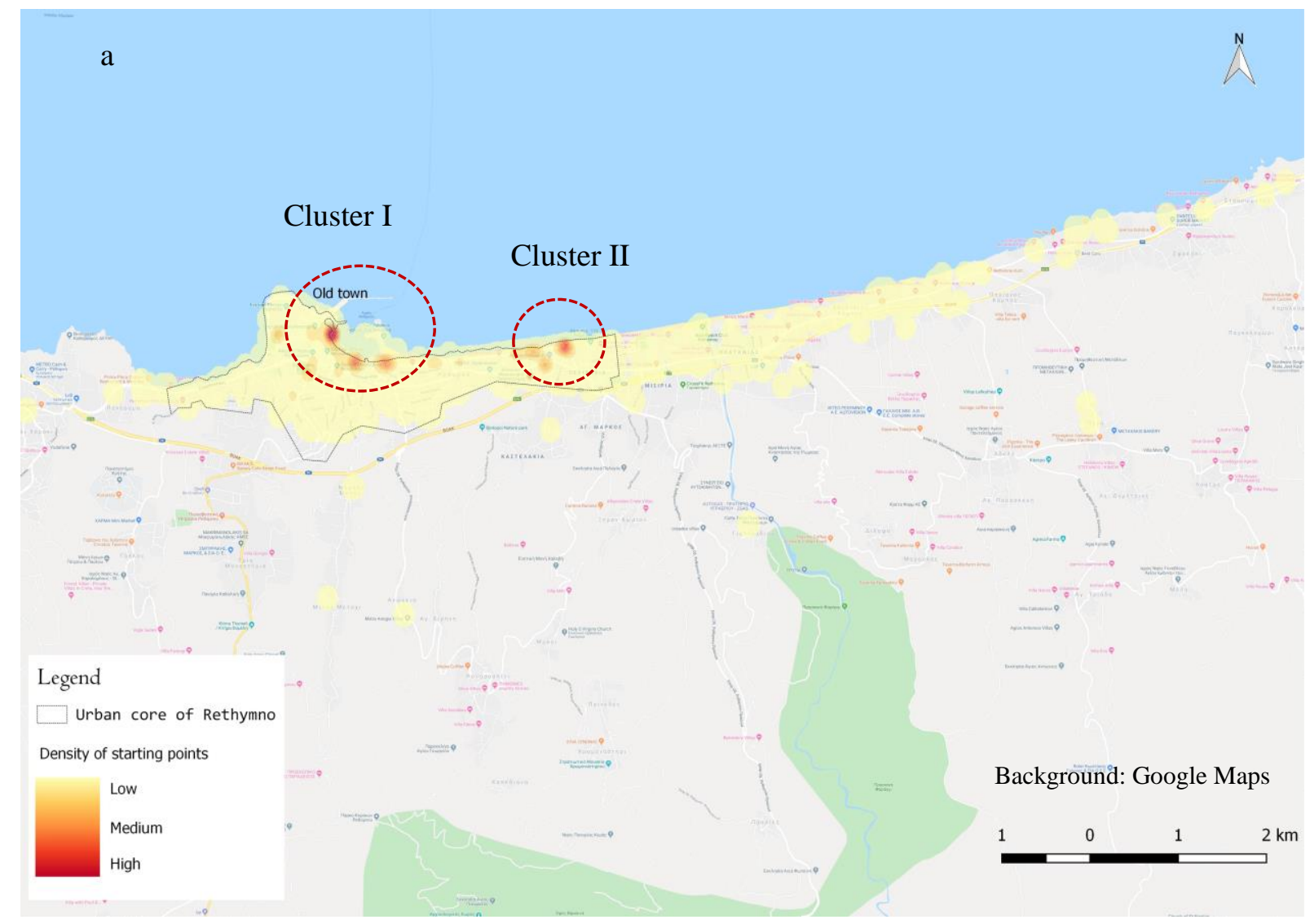




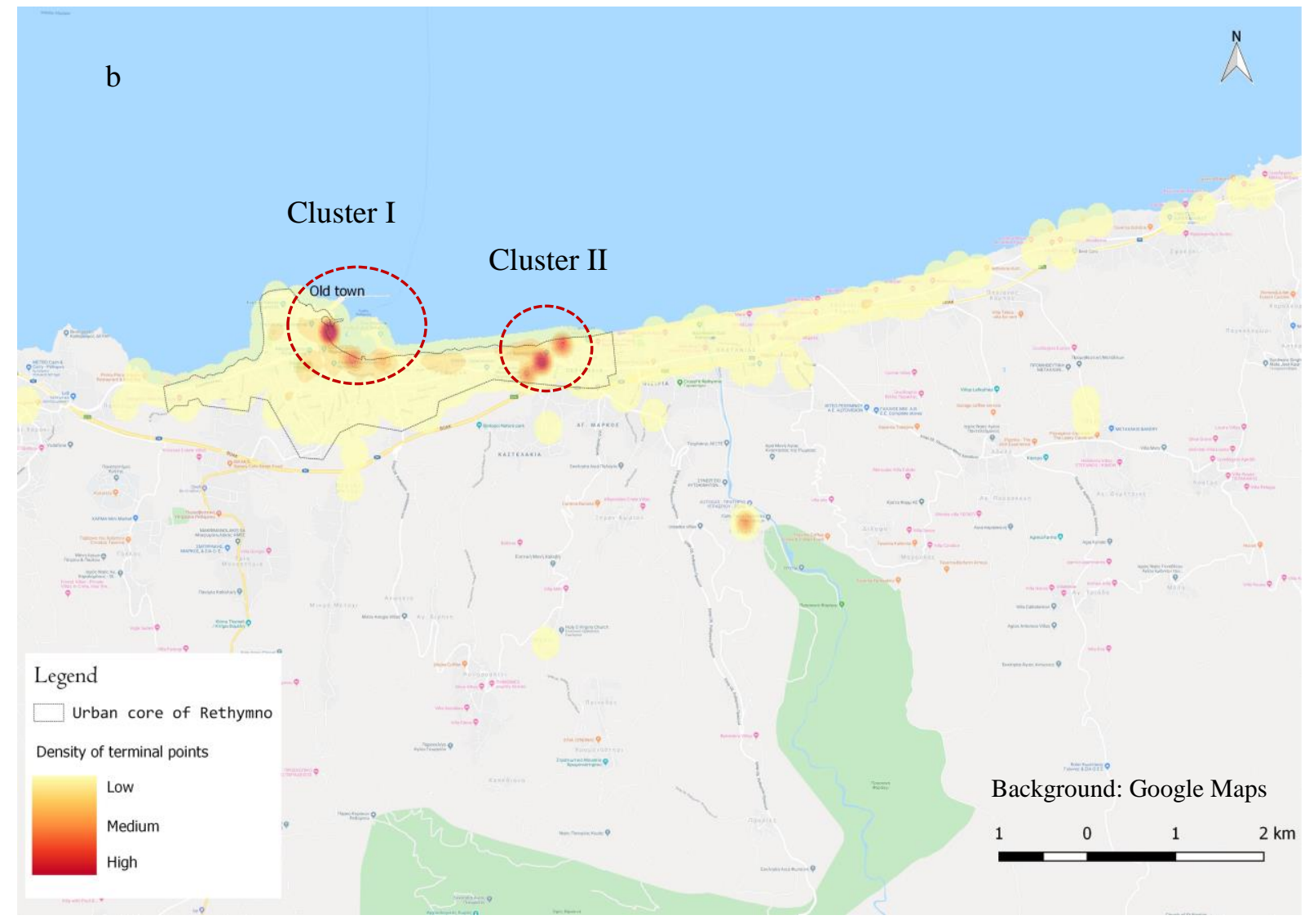

Figure 5: Kernel Density of Starting (a) and Terminal (b) points of the preferred routes

\subsection{Users' Perceptions about the DBSS Operation}

The second pillar of the present analysis includes the interpretation of a brief questionnaire answered by 534 DBSS users. The total number of subscriptions by $28^{\text {th }}$ February 2019 as recorded by the operator was 2123 ; thus the survey captured $25.2 \%$ of the total sample. The seven-item survey aimed to capture the users' perceptions reflecting and affecting their experience of the first ever implementation of a DBSS in Greece. The first question of the questionnaire was about the frequency of its use, helping us to see how regularly scheme subscribers use the scheme. This may indicate to an extent the level of integration of DBSS in the overall transport system of the city. Figure 6 presents the frequency with which residents and visitors use the system. 


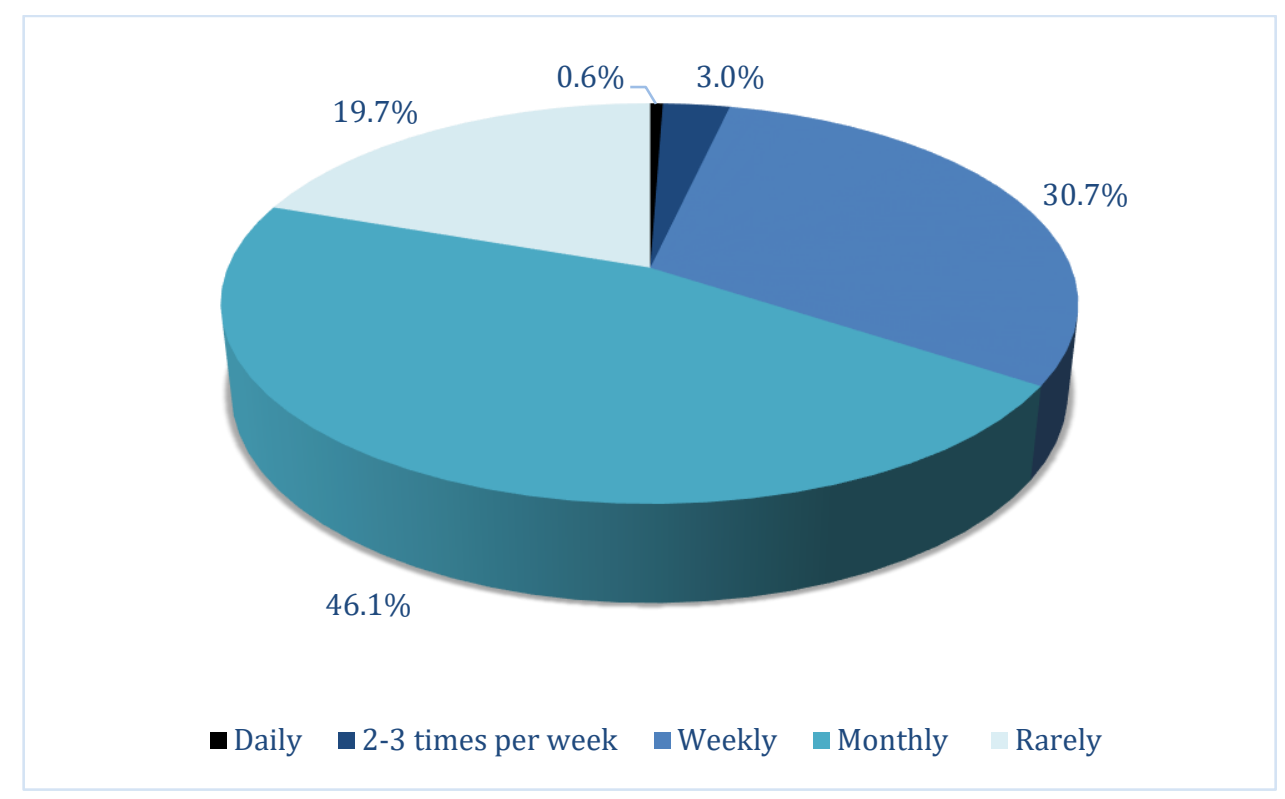

Figure 6: Frequency of use

It is clear that the majority of users rely on the DBSS to support their commuting or recreation in a monthly basis (46.1\%). Around 30\% of the travelers filling in the survey use the system in a weekly basis. There are significantly less, travelers that could be classified as loyal users (i.e. those using the DBSS on a daily basis or 2-3 times per week); their shares among the respondents was $0.6 \%$ and $3 \%$ respectively. Finally, a fifth of the system's users represent rare users who cannot develop a strong and consistent usage relation with this particular means of transport. Classifying the users into frequent users (usage rate at least weekly) and occasional ones (monthly use at best), adds up to the following shares: the frequent users and occasional users represent respectively $34.3 \%$ and $65.7 \%$ of the total sample.

We asked the users to assess from a pool including eight possible answers as this has been informed by previous works (i.e. Nikitas et al. 2016; Nikitas, 2018) the potential reasons that might deter their use of DBSS. The respondents had the choice to choose as many options as they would think were suitable. Figure 7 presents the respective results of the frequent and the occasional users, separately, because of statistically significant differences between the two groups. 


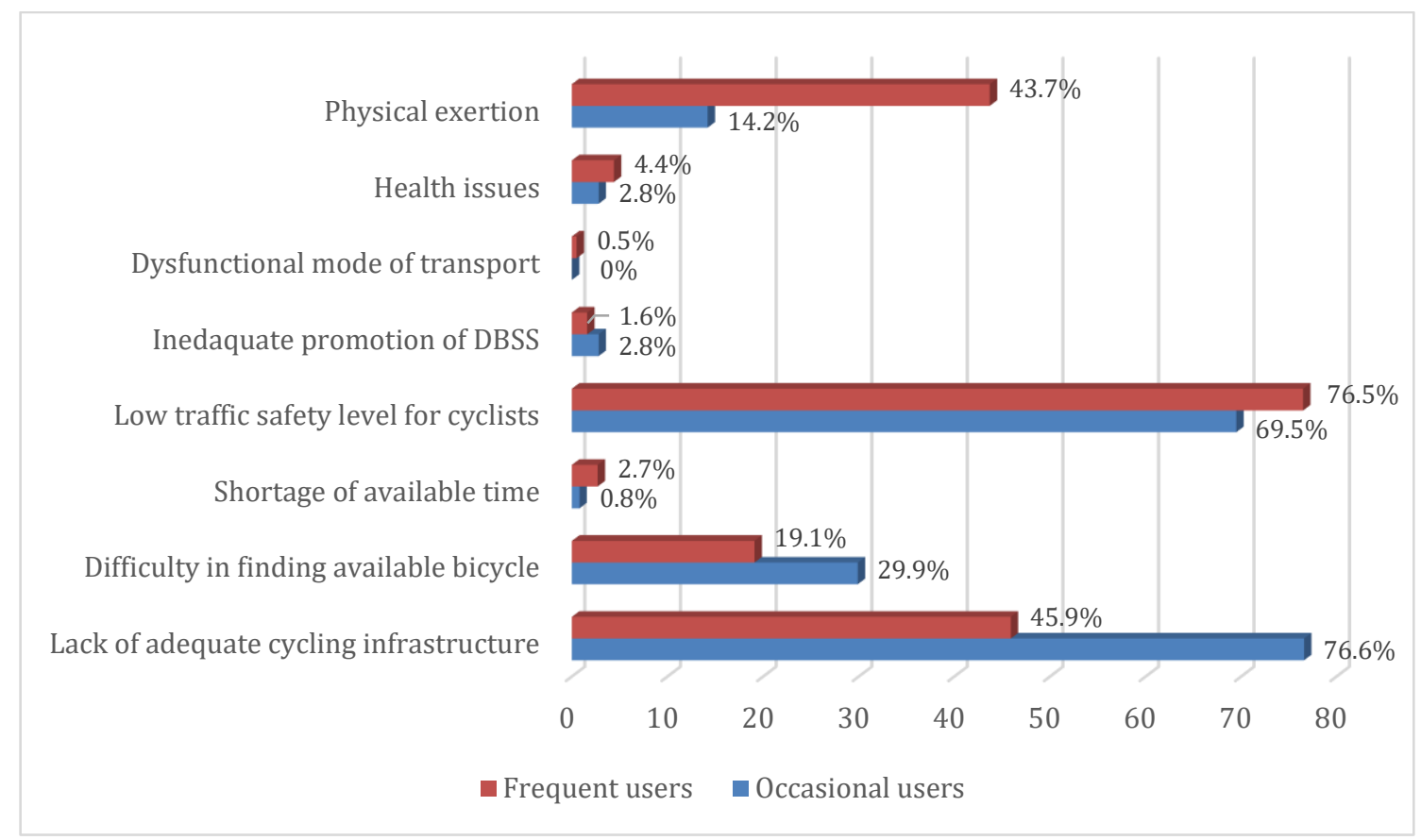

Figure 7: Barriers that could result in limiting the use of DBSS

The most critical reason for not using more regularly the local DBSS for frequent users is their perception that cyclists are exposed to low traffic safety followed by the lack of adequate cycling infrastructure in Rethymno. Also, more than four in every ten frequent users promote as a reason for not using the scheme more often physical exertion, although the bikes are pedal assisting. This perhaps indirectly denotes that pedal assisting systems do not significantly change the user experience and perceptions reflecting how physically demanding cycling could be as a mode of active transportation. Another interesting finding referring to this answer that is supported by our observational field work is that frequent users used the bikes primarily as normal bikes thinking that the electric support they offer is cheating. This is in line with the findings of Jones et al. (2016).

In the case of occasional users, similar reasons are noted regarding their reluctance in using the DBSS, with the lack of cycling infrastructure being the most important reason followed by the belief that traffic safety for cyclists is low. However, there is a very big percentage difference between the two groups in the lack of cycling infrastructure issue; more frequent bike share users are not as likely to see it a barrier. Occasional users were not as likely to report physical exertion as deterrent probably because they cycle less and more likely to say that finding a bike could be difficult. Most of these reasons making users reluctant to increase the regularity with which they embrace the scheme are external to the local DBSS operation specifics, meaning that users feel satisfied by the existing efficiency of the system per se.

The third part of the questionnaire consists of five questions regarding scheme-specific acceptance and the role of the DBSS for the city. The possible answers to these questions are yes and no. More complicated 5-point Likert scale-based questions (and a longer survey as a whole) were piloted before the launch of the data collection but were unsuccessful; the response rates were very low since the finite pool of respondents did not want to invest a lot of time to this research exercise. The approach chosen helped us collect 534 responses which makes our results entirely representative of the total user population and provides an extra layer of validity. 
The first of these questions looks into the cost of the system. The vast majority of the respondents stated that the scheme is affordable for the user. The second refers to the role of the DBSS as an effective solution to tackle traffic congestion. Many users do not consider it a factor that can relieve the city from traffic congestion. The next question deals with the complexity of the system. Noticeably, the vast majority of the sample mentioned that the DBSS is simple and easy to use. The fourth question refers to whom the DBSS is suitable for. Both occasional and frequent users agree that it is not solely for tourists, but on the contrary it can constitute a means of transport valuable to permanent residents. The last question refers to the appropriateness of the investment for Rethymno, where the majority of both occasional and frequent users answered that the DBSS is not an effective investment for the city. This is explained through the key argument that appropriate infrastructure (cycling lanes, traffic calming, extended bike parking facilities) should have preceded the establishment of DBSS; this would have made the DBSS investment timelier and more meaningful.

In general terms, the results of this short survey show that the majority of users considers the DBSS as beneficial and functional but in need to be part of a broader pro-cycling investment effort to reach its potential. Therefore, improving its operation and expanding the scheme can possibly further promote sustainable mobility and especially cycling.

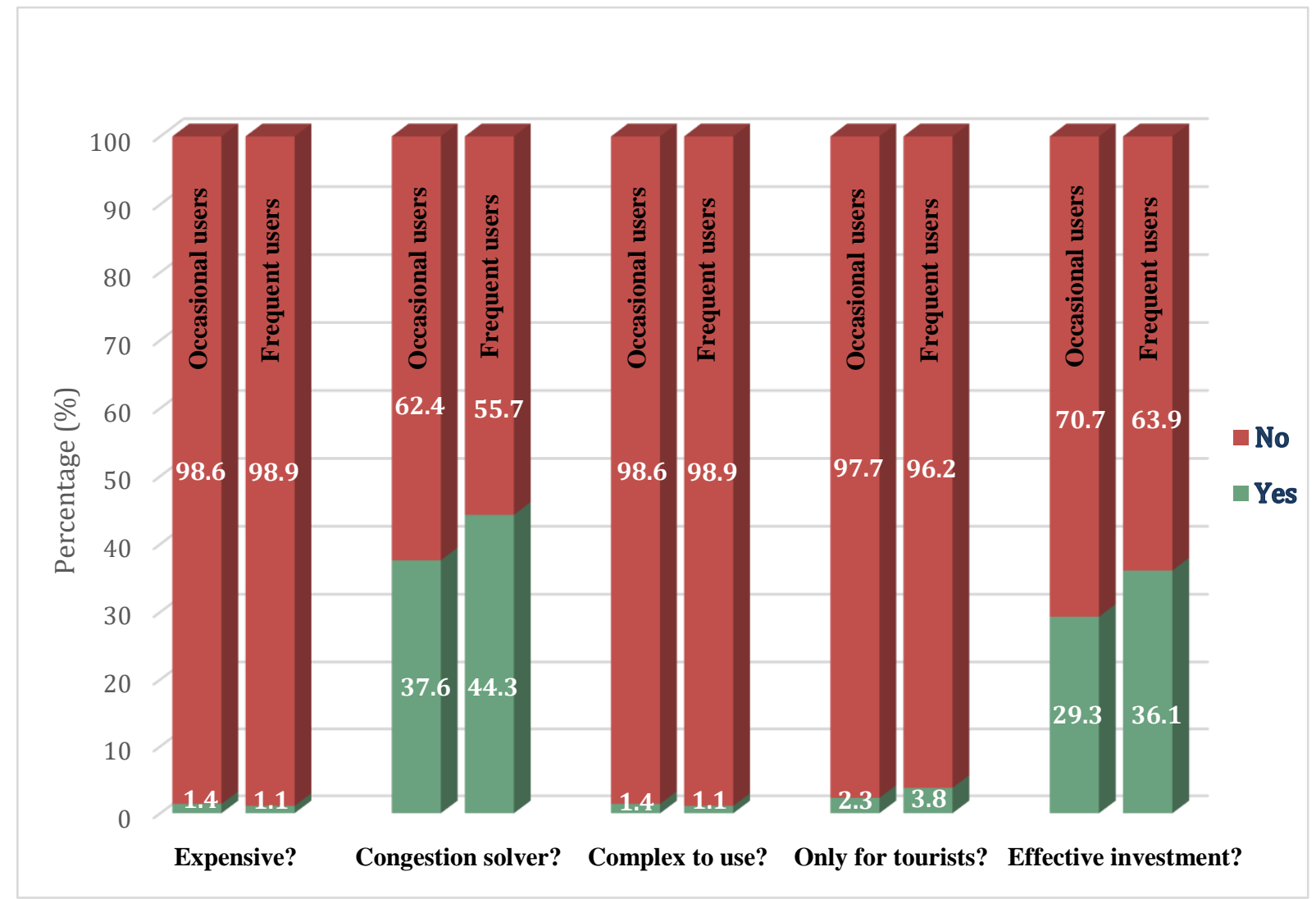

Figure 8: User perceptions about DBSS

Further to the descriptive analysis, a statistical model (presented in Table 2) was developed to describe and quantify the relationship between frequency of DBSS usage and variables representing perceived barriers to usage, congestion relief potential and effectiveness of financial investment. Binomial regression was employed since it is an appropriate generic model for the empirical analysis of any binary dependent variable, which in this case represented the frequency of using DBSS in Rethymno. Various models were tested, using a combination of independent variables, but only the best fit, a model referring to statistically 
significant results, is presented. It should be noted that because the sample's overwhelming perceptions that DBSS is easy to use and appropriate for both tourist and local populations these two possible independent variables were not used in the model.

Table 2: Evaluation of DBSS perceptions

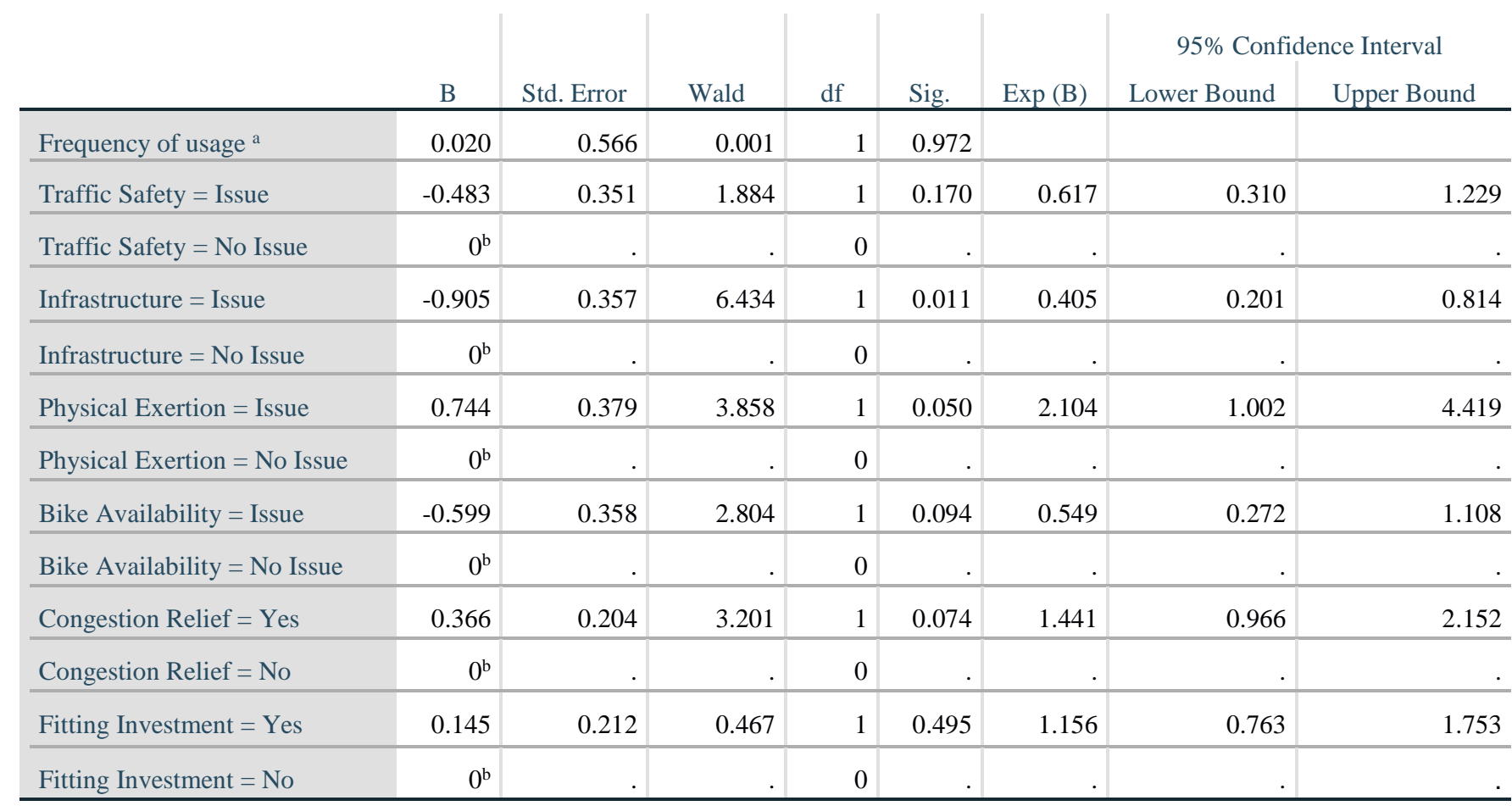

$\mathrm{N}=534$, Model chi-square $=66.107 ; \mathrm{p}<0.01,-2 \log$ likelihood $=98.480$, Nagelkerke Pseudo $\mathrm{R}^{2}=0.161$.

a. The reference category is occasional users.

b. This parameter is set to zero because it is redundant.

The model confirms statistical significance for the relationships between usage frequency and perceived lack of cycling infrastructure $(\mathrm{p}<0.05)$, physical exertion (for $\mathrm{p} \leq 0.05$ ), bike availability $(\mathrm{p}<0.1)$ and congestion relief $(\mathrm{p}<0.1)$. The frequency of usage relationships with traffic safety concerns and investment appropriateness do not appear to be statistically significant for this model at least. Our results specifically show that the odds of people believing that cycling infrastructure is lacking or thinking that bikes are not always available to use Bikeazy are lower than people that think otherwise. The odds of people considering that bicycling needs considerable physical effort or perceiving DBSS as an intervention creating congestion relief to use Bikeazy are higher than people with different opinions on these matters.

\section{Discussion}

The present work develops a thorough empirical and theoretical understanding of the first Greek DBSS' usage and acceptance norms. It specifically contributes to the state of art by: a) analysing current usage patterns through the use of real trip data, b) examining the various reasons that deter the use of the system and c) exploring some of the key user perceptions affecting and reflecting usage of DBSS. Our results meant to provide best practice guidance to shared and active mobility providers and transport policy-makers looking to introduce or improve their dockless fleets.

Our findings indicate that the system has a considerable number of users already in its first year of operation, at least for a city that has yet to establish a pro-cycling ethos. We suggest that the 
fleet size is adequate but the scheme may be somewhat underused for now. The latter is not very uncommon; local scheme usage rates in many cities remain below expectations (Nikitas 2019; Wang et al., 2018b; Zhang et al., 2015) even when a scheme has many subscribers. The system is used primarily for short distance trips but in few cases may be a complementary mode for trips of greater distance. This is in line with research suggesting that bike sharing's peak in modal share comes for trips less than 0.5 miles (de Nazelle et al., 2010) and acknowledging the role of bike sharing as a first- or last-mile solution for longer trips (Ma et al., 2019; Qiu \& He, 2018; Yang et al., 2019; Zhang et al., 2019). Also, likewise with Caufield et al. (2017) that looked at a similarly sized city, trip times are in average very short.

Rethymno's DBSS was found to serve the whole urban core area of the city and its main suburbs, but the highest supply and demand arises in the city centre and the eastern part of the urban core (close to the seafront with many touristic and commercial activities). This system placement settings and respective demand and supply norms have been observed in the majority of cities with local bike sharing schemes (El-Assi et al., 2017; Vogel et al, 2011). The literature suggests that peripheral stations, or less central pick up and drop off locations for the case of DBSS, are used less frequently (Caspi \& Noland, 2019). Possibly one recommendation for Bikeazy would be about the need to achieve a better distribution of the bikes within the city so that all bikes carry similar loads.

In terms of usage uptake the system is used primarily in an occasional basis; frequent users are significantly fewer than those self-reporting a less regular bike sharing activity. This is very typical even for municipalities with far more established pro-cycling identities like Gothenburg, Sweden (Nikitas et al., 2016) or Vancouver, Canada (Winters et al., 2019). This however highlights the massive untapped potential of the scheme in usage terms and the need to become an even more attractive choice to existing and potential subscribers.

The main barriers making users reluctant to use bike sharing services in a more frequent basis are low traffic safety and lack of cycling infrastructure for both frequent and occasional users. This is in line with many studies trying to identify reasons that could reduce bike share usage and uptake (e.g. Fishman et al., 2015; Nikitas 2018; Nikitas et al., 2016). Rethymno is in advantageous position however, since adverse weather conditions (El-Assi et al., 2017; Sun et al., 2018) or compulsory helmet use regulations (Ahillen et al., 2016; Fishman et al., 2012) that have been restricting access to other bike sharing schemes across the world are not of concern. Our respondents believe that appropriate infrastructure (cycling lanes, traffic calming measures, bike parking and integrating cycling with the bus system) should have preceded the investment of a local DBSS. The work of Pucher \& Buehler (2008) actually confirms that broader pro-bike investments may be a prerequisite for high bike share usage rates so Rethymno's DBSS cannot reach its potential without this type of support. Increasing perceived and actual traffic safety for cyclists through educating cyclists and drivers or segregating bicycling from motor traffic is considered an effective way to promote bike sharing (Liu et al., 2012). Scheme expansion has been also recorded as a successful strategy for increasing bike share usage in larger cities and schemes (Zhang et al., 2016). Strategic investments on traffic safety improvements and bike-friendly infrastructure should thus improve the uptake and usage rates of the system studied. However, these may not be enough on their own; continuous participatory exchange with the public and close collaboration with the local authorities are equally important for the long-term sustainability of a newly installed DBSS according to lessons learnt by Manchester Mobike's failure in a span of 15 months (Dudley et al., 2019; Nikitas, 2019).

Frequent users also reported reluctance to use more regularly the DBSS because of physical exertion concerns despite the pedal-assisting nature of the offered bikes. This means that pedal assistance mechanisms cannot alter perceptions and improve user experience regarding the amount of physical effort needed for cycling. So, despite the significant extra cost that the electric capacity of the fleet means to the Rethymno DBSS the users still do not see it being as 
effortless as the car is. However, in line with Dill \& Rose (2012), at least in a longer-term horizon this system's capacity could help expanding the local bike sharing market. The results of our study perhaps indirectly indicate that the Bikeazy bicycles are not used by many regular users in their full electrical capacity; this could be due to ignorance or due to the preference for non-assisted bicycling that supports health, exercise and sustainability needs. Our field observations confirm that some of the users probably did not fully understood or wanted to take advantage of the pedal-assisting ability of the Bikeazy fleet and also that there is some competition between the DBSS and the electrical scooter system especially for the people seeking to get electromobility services.

Users consider, almost universally, that the DBSS is an affordable and easy-to-use travel option. A bike sharing scheme's affordability has a significant positive association with new trips (Buehler \& Hamre, 2014; Ricci, 2015) so the low charges of Rethymno's DBSS could help it to be successful long-term. Ease of use, including ease of signing up to a programme, has been also found to consistently increase usage rates (Fishman et al., 2012). Our respondents unanimously agreed that the scheme is suitable for both locals and tourists. This is important because Rethymno needs DBSS to play a role beyond being a tourist travel mode; it should have the capacity to provide a long-term commuting mechanism and be a modal shift generator as described by best practice guidance (Jain et al., 2018 and Barbour et al., 2019 respectively). At the same being one of Europe's top tourist destinations that faces a need for increasing travel supply without generating extra motor traffic during summer months and knowing that 'tourists are very interested in using bike sharing frequently and for multiple purposes' (Kaplan et al., 2015) means that the scheme's suitability for tourists is a key asset for its continuous improvement.

Our respondents, despite empirical evidence suggesting that the availability of bike sharing reduces traffic congestion in neighbourhood level (Hamilton \& Wichman, 2018) and during peak times (Wang \& Zhou, 2017), were split when asked about DBSS' potential to solve traffic congestion problems with more of them being negative. Those using the scheme more regularly however were more likely to see that bike sharing can make a positive difference. Education and promotion mechanisms about this particular quality of bike sharing should be adopted by the scheme providers to highlight the positive contribution that the scheme could make.

The rather controversial result about the respondents' disbelief of the system's suitability as an effective local financial investment reflects some of the problems that are highlighted by this research and are not related to the system per se as all the positive indicators listed in this discussion denote. These problems clearly refer to perceptions about limited traffic safety provision for cyclists and to a lack of sufficient pro-bike infrastructure and culture regarding the city. This disbelief, that was associated to some degree with usage frequency, is potentially reversible when the scheme matures and the city improves its overall bicycling provision.

With the increased attention that cycling is receiving as a means of addressing urban transport problems and with the fruits of the consistent work on its development being evident in cities across the globe (Parkin, 2015), bike sharing, much like proposed by Tuama (2015), should be used, in Rethymno and other municipalities, as a tool giving rise to a more bike-friendly city for a potentially much broader demographic. This is because bike sharing can help normalising the image of cycling as an everyday activity and encourage modal shift (Goodman \& Chesire, 2014), something that will indirectly lead to a much broader positive impact than what the isolated bike sharing usage reflects. 


\section{Strengths, Limitations and Future Studies}

The high value of this study is related to its timing as it is the first to examine and present the case of DBSS in Greece. It is based on three methods (i.e. secondary data analysis, observations and questionnaires) and thus offers validation and triangulation merits that add to the breadth and depth of a research (Johnson et al., 2007). The survey sample is entirely representative of the user population since it captures the attitudes of 534 respondents out of 2123 total subscribers that include many one-off users and visitor passes; an outreach so successful is rather rare. Access to secondary data referring to the daily usage of a scheme are usually notoriously difficult to get for most bike sharing schemes as practice shows and this is one of the few available studies that have achieved that.

However, it should be acknowledged that the present study suffers from some limitations that could have influenced a few of the findings to some degree. The usage data exploited for the secondary data analysis were collected and provided by the private operator running the scheme and not by the authors thus the accuracy of the data has not passed our own data collection control practices. The self-report data, collected in the survey phase, may be subject to numerous biases and to inaccuracies (Paulhus, 1991), especially since these primarily refer to attitudes for a scheme with a seemingly strong pro-social profile (Nikitas, 2018). This could mean that "accepting" bike sharing might have been "the right answer" for some respondents, thus we do acknowledge the existence to some degree of social desirability bias. Gender and other important socio-demographic information were not recorded in the survey since our priority was to make this shorter, uncomplicated, more anonymous and thus more answerable. This might have affected negatively our explanatory power in justifying and interpreting some of our results. For example, in Section 5.2 it is suggested that "physical exertion" is one of the key issues that may deter bike share usage. This result could have been influenced by gender (i.e. male and female may have a varied opinion on "physical exertion" and "pedal-assisting") in light of the fact that gender differences have been found in many bike sharing schemes (Beecham \& Wood, 2014). Also, the work cannot provide a definite answer to the question 'what mode of transport did DBSS trips replace?' due to lack of relevant data. Based on our observations and the short duration of the trips analysed our guesstimate is that that bike sharing is used mainly as a last- and first-mile solution and reduced primarily short car trips and perhaps to a lesser extent bus trips and walking.

Future research in Rethymno's Bikeazy, including non-user populations too, that will eventually provide a longitudinal dimension to this body of work are planned from the authors. Sister mixed-method studies looking to capture bike sharing attitudes, travel patterns and user experiences are scheduled for other bike sharing schemes located primarily in Greece.

\section{Conclusions}

This paper reports a mixed-method study of Greece's first DBSS that it is also its largest local BSS. The research develops an analysis framework for a medium-sized city that is not limited only to the observed characteristics (e.g. routes or rents) but examines perceptions reflecting and affecting the current user experience of 534 service subscribers. More specifically, the research provided evidence that the newly founded DBSS of Rethymno has a business model that promises to work if supported properly by the local authorities. This support should come in the form of complementary pro-cycling investments that address problems mainly referring to traffic safety concerns and lack of cycling infrastructure that make, at present, some people to think that DBSS may be "too much" for the city. Investments in awareness, education and public engagement exercises so that people learn about the scheme and its full potential are also of critical importance for improved acceptance and usage rates. It should be acknowledged however, that this is not a particularly straightforward task for a part of the world that has not 
yet established, with few exceptions like the city of Trikala in North Greece, a bike-friendly culture. It will take time and combined effort by the scheme providers and transport authorities to make this scheme a mainstream choice for more people. Tourists that might have been more accustomed to bike share in their own countries, may be targeted by the DBSS providers as a more accessible market group who can potentially play a critical role in inspiring and propagating the message of modal shift for everyday commuters. Locals interested in recreational cycling could be another market group of particular importance because users overwhelmingly recognised in the survey that DBSS is meaningful for the resident population too. The results of the study can directly inform the future development of the system per se but also inspire local planners to take on other micromobility interventions. The lessons learnt may also be valuable in wider efforts, for the context at least of similar small and medium-sized cities, which are looking to develop a more sustainable mobility ethos in societies that are in the very first stage of being introduced to shared and active transport initiatives.

\section{Acknowledgements}

We would like to express our deepest gratitude to Dimitris Dimitriadis and George Voulgaroudis from Brainbox S.A. (i.e. the local bike sharing provider) that gave us access to their database and supported the overall data processing.

\section{References}

Acquier, A., Daudigeos, T., \& Pinkse, J. (2017). Promises and paradoxes of the sharing economy: An organizing framework. Technological Forecasting and Social Change, $125,1-10$.

Ahillen, M., Mateo-Babiano, D., \& Corcoran, J. (2016). Dynamics of bike sharing in Washington, DC and Brisbane, Australia: Implications for policy and planning. International Journal of Sustainable Transportation, 10(5), 441-454.

Athanasopoulos, K., \& Vlastos, T. (2015). Integrated Urban and Transport Planning in touristic areas aiming at Sustainable Mobility. The case of Rethymno. Proceedings of the 7th International Congress on Transportation Research. Athens, Greece .

Attard, M., \& Shiftan, Y. (Eds.). (2015). Sustainable Urban Transport. Emerald Group Publishing.

Bakogiannis, E., Kyriakidis, C., Siti, M., \& Eleftheriou, V. (2017). Four stories for sustainable mobility in Greece. Transportation Research Procedia, 24, 345-353.

Bakogiannis, E., Vassi, A., Siti, M., \& Christodoulopoulou, G. (2016). Developing a sustainable mobility plan in Piraeus with special emphasis on cycling. Journal of Traffic and Transportation Engineering, 4, 61-74.

Banister, D. (2008). The sustainable mobility paradigm. Transport Policy, 15 (2), 73-80.

Barbour, N., Zhang, Y., \& Mannering, F. (2019). A statistical analysis of bike sharing usage and its potential as an auto-trip substitute. Journal of Transport \& Health, 12, 253262.

Beecham, R., \& Wood, J. (2014). Exploring gendered cycling behaviours within a large-scale behavioural data-set. Transportation Planning and Technology, 37(1), 83-97.

Brueckner, J. (2000). Urban Sprawl: Diagnosis and Remedies. International Regional Science Review, 23 (2) , 160-171.

Buehler, R., \& Hamre, A. (2014). Economic benefits of capital bikeshare: A focus on users and businesses (No. VT-2013-06). Mid-Atlantic Universities Transportation Center.

Caspi, O., \& Noland, R. B. (2019). Bikesharing in Philadelphia: Do lower-income areas generate trips?. Travel Behaviour and Society, 16, 143-152. 
Caulfield, B., O'Mahony, M., Brazil, W., \& Weldon, P. (2017). Examining usage patterns of a bike-sharing scheme in a medium sized city. Transportation Research Part A: Policy and Practice, 100, 152-161.

Craig, B., Brereton , F., \& Bailey, S. (2017 ). The economic contribution of public bike-share to the sustainability and efficient functioning of cities . Sustainable cities and society, $28,76-87$.

Davis, L. (2014 ). Rolling along the last mile: Bike-sharing programs blossom nationwide . Planning, 80(5), 10-16.

DeMaio, P. (2009 ). Bikesharing: History, Impacts, Models of Provision and Future . Journal of Public Transportation, 12(4), 41-56.

de Chardon, C. M. (2019) The contradictions of bike-share benefits, purposes and outcomes. Transportation Research Part A: Policy and Practice, 121, 401-419.

de Nazelle, A., Morton, B. J., Jerrett, M., \& Crawford-Brown, D. (2010). Short trips: an opportunity for reducing mobile-source emissions?. Transportation Research Part D: Transport and Environment, 15(8), 451-457.

Dill, J., \& Rose, G. (2012). Electric bikes and transportation policy: Insights from early adopters. Transportation Research Record, 2314(1), 1-6.

Dudley, G., Banister, D., \& Schwanen, T. (2019). The dynamics of public participation in new technology transitions: the case of dockless bicycle hire in Manchester. Built Environment, 45(1), 93-111.

Duran-Rodas, D., Chaniotakis, E., \& Antoniou, C. (2019). Built Environment Factors Affecting Bike Sharing Ridership: Data-Driven Approach for Multiple Cities. Transportation Research Record, 0361198119849908.

El-Assi, W., Mahmoud, M. S., \& Habib, K. N. (2017). Effects of built environment and weather on bike sharing demand: a station level analysis of commercial bike sharing in Toronto. Transportation, 44(3), 589-613.

ELSTAT (2011). Greek Census Report of Population and Buildings. Piraeus, Greece.

Fishman, E. (2016). Bikeshare: a review of recent literature . Transport Reviews, 36(1) , 92113 .

Fishman, E., \& Schepers, P. (2014 ). Global bike share: What the data tells us about safety. Paper presented at the International Cycling Safety Conference . Gothenburg, Sweden.

Fishman, E., Washington, S., Haworth, N., \& Watson, A. (2015). Factors influencing bike share membership: An analysis of Melbourne and Brisbane. Transportation Research Part A: Policy And Practice, 71, 17-30.

Fishman, E., Washington, S., \& Haworth, N. (2014 ). Bike share's impact on car use: Evidence from the United States, Great Britain and Australia . Transportation Research Part D: Transport and Environment, 31 , 13-20.

Fishman, E., Washington, S., \& Haworth, N. (2013 ). Bike share: A synthesis of the literature. Transport Reviews, 33(2), 148-165.

Fishman, E., Washington, S., \& Haworth, N. (2012). Barriers and facilitators to public bicycle scheme use: A qualitative approach. Transportation Research Part F: Traffic Psychology and Behaviour, 15(6), 686-698.

Goodman, A., \& Cheshire, J. (2014). Inequalities in the London bicycle sharing system revisited: impacts of extending the scheme to poorer areas but then doubling prices. Journal of Transport Geography, 41, 272-279.

Gossling, S., \& Choi, S. (2015 ). Transport transitions in Copenhagen: Comparing the cost of cars and bicycles . Ecological Economics, 113 , 106-113.

Gudmundsson, H. (2004). Sustainable transport and performance indicators . In R. Hester, \& R. Harrison, Transport and the Environment-Issues in Environmental Science and Technology, 20 (pp. 35-63). Cambridge, UK: Royal Society of Chemistry. 
Hamari, J., Sjöklint, M., \& Ukkonen, A. (2016 ). The Sharing Economy: Why People Participate in Collaborative Consumption. Journal of the Association for Information Science and Technology, 67(9), 2047-2059.

Hamilton, T. L., \& Wichman, C. J. (2018). Bicycle infrastructure and traffic congestion: Evidence from DC's Capital Bikeshare. Journal of Environmental Economics and Management, 87, 72-93.

Jabareen, Y. (2006). Sustainable urban forms: their typologies, models and concepts. Journal of Planning Education and Research, 26 (1), 38-52.

Johnson, R. B., Onwuegbuzie, A. J., \& Turner, L. A. (2007). Toward a definition of mixed methods research. Journal of Mixed Methods Research, 1(2), 112-133.

Jones, T., Harms, L., \& Heinen, E. (2016). Motives, perceptions and experiences of electric bicycle owners and implications for health, wellbeing and mobility. Journal of Transport Geography, 53, 41-49.

Kaplan, S., Manca, F., Nielsen, T. A. S., \& Prato, C. G. (2015). Intentions to use bike-sharing for holiday cycling: An application of the Theory of Planned Behavior. Tourism Management, 47, 34-46.

Li, X., Zhang, Y., Sun, L., \& Liu, Q. (2018). Free-floating bike sharing in jiangsu: Users' behaviors and influencing factors. Energies, 11(7), 1664.

Li, Y., Zhu, Z., \& Guo, X. (2019). Operating Characteristics of Dockless Bike-Sharing Systems near Metro Stations: Case Study in Nanjing City, China. Sustainabaility, 11 (8) , 2256.

Liu, Z., Jia, X., \& Cheng Wen. (2012). Solving the Last Mile Problem: Ensure the Success of Public Bicycle System in Beijing. Procedia - Social and Behavioral Sciences, 43 , 7378 .

Luo, H., Kou, Z., Zhao, F., \& Cai, H. (2019). Comparative life cycle assessment of stationbased and dock-less bike sharing systems. Resources, Conservation and Recycling, 146, 180-189.

Ma, X., Zhang, X., Li, X., Wang, X., \& Zhao, X. (2019). Impacts of free-floating bikesharing system on public transit ridership. Transportation Research Part D: Transport and Environment, 76, 100-110.

Manzi, G., \& Saibene, G. (2018 ). Are they telling the truth? Revealing hidden traits of satisfaction with a public bike-sharing service. International Journal of Sustainable Transport, 12 , 253-270 .

Marshall, S. (2005). Streets \& Patterns. Abbingdon: Spon Press.

Midgley, p. (2011 ). Bicycle Sharing Schemes: Enhancing sustainable mobility in urban areas. New York : Commision on Sustainable Development, Nineteenth.

Mooney, S., Hosford, K., Howe, B., Yan, A., Winters, M., Bassok, A., \& Hirsch, J. (2019). Freedom from the station: Spatial equity in access to dockless bike share. Journal of Transport Geography, 74, 91-96.

Morton, C. (2018). Appraising the market for bicycle sharing schemes: Perceived service quality, satisfaction, and behavioural intention in London. Case Studies on Transport Policy, 6(1), 102-111.

Newman, P. W. and Kenworthy, J. R. (1996) The land use - transport connection: An overview. Land Use Policy, 13(1), 1-22

Nieuwenhuijsen, M., \& Khreis, H. (2016). Car free cities: Pathway to healthy urban living. Environmental International, 94, 251-262.

Nikitas, A. (2018). Understanding bike-sharing acceptability and expected usage patterns in the context of a small city novel to the concept: A story of 'Greek Drama'. Transportation Research Part F:Ttraffic Psychology and Behaviour, 56, 306-321. 
Nikitas, A., Wallgren, P., \& Rexfelt, O. (2016). The paradox of public acceptance of bike sharing in Gothenburg. Proceedings of the Institution of Civil Engineers-Engineering Sustainability, 169 (3), 101-113.

Nikitas, A. (2019) How to Save Bike-Sharing: An Evidence-Based Survival Toolkit for Policy-Makers and Mobility Providers. Sustainability, 11(11), 3206.

Nikitas, A., Wang, J. Y., \& Knamiller, C. (2019). Exploring parental perceptions about school travel and walking school buses: A thematic analysis approach. Transportation Research Part A: Policy and Practice, 124, 468-487.

O'Tuama, D. (2015). Ripples through the city: Understanding the processes set in motion through embedding a public bike sharing scheme in a city. Research in Transportation Business \& Management, 15, 15-27.

Pal, A., \& Zhang, Y. (2017 ). Free-floating bike sharing: solving real-life large-scale static rebalancing problems . Transportation Research Part C: Emerging Technologies, 80 , 92-116.

Parkes, S., Marsden, G., Shaheen, S., \& Cohen, A. (2013 ). Understanding the diffusion of public bikesharing systems: evidence from Europe and North America. Journal of Transport Geography, 31 , 94-103.

Parkin, J. (2015). Editorial for themed issue on 'Managing the business of cycling'. Research in Transportation Business and Management, 15, 1-5.

Paulhus, D. L. (1991). Measurement and control of response bias. In Robinson, J. P., Shaver P. R. and Wrightsman L. S. (ed.), Measures of Personality and Social Psychological Attitudes, San Diego, CA: Academic Press.

Pucher, J., \& Buehler, R. (2008). Making cycling irresistible: lessons from the Netherlands, Denmark and Germany. Transport reviews, 28(4), 495-528.

Pucher, J., Dill, J., \& Handy, S. (2010). Infrastructure, programs, and policies to increase bicycling: an international review. Preventive Medicine, 50, S106-S125.

Pucher, J., \& Buehler, R. (2012 ). City Cycling . Cambridge, MA: MIT Press.

Qiu, L. Y., \& He, L. Y. (2018). Bike sharing and the economy, the environment, and healthrelated externalities. Sustainability, 10(4), 1145.

Rahim Taleqani, A., Hough, J., \& Nygard, K. (2019). Public opinion on dockless bike sharing: A machine learning approach. Transportation Research Record: Journal of the Transportation Research Board, 1-10.

Reiss, S., Paul, F., \& Bogenberger, K. (2015). Empirical analysis of Munich's free-floating bike sharing system: GPS-booking data and customer survey among bikesharing users. Proceedings of Transportation Research Board 94th Annual Meeting, (p. 3741). Washington DC, United States.

Ricci, M. (2015 ). Bike sharing: A review of evidence on impacts and processes of implementation and operation . Research in Transportation Business and Management.

Shaheen, S., \& Guzman, S. (2011 ). Worldwide bikesharing. ACCESS Magazine, 1(39), 2227.

Shaheen, S., Cohen, A., \& Martin, E. (2013 ). Public Bikesharing in North America . Transportation Research Record, Journal of Transportation Research Board, 2387 , 83-92.

Shaheen, S., Guzman, S., \& Zhang, H. (2010). Bike sharing in Europe, the Americas and Asia . Transportation Research Record, Journal of the Transportation Research Board, 2143 , 159-167.

Shaheen, S., Guzman, S., \& Zhang, H. (2012). Bikesharing across the Globe. In J. Pucher, \& R. Buehler, City Cycling. Cambridge, MA: MIT Press.

Shen, Y., Zhang, X., \& Zhao, J. (2018). Understanding the usage of dockless bike sharing in Singapore. International Journal of Sustainable Transportation, 12(9), 1-15. 
Shi, J.-g., Si, H., Wu, G., Su, Y., \& Lan, J. (2018). Critical Factors to Achieve Dockless BikeSharing Sustainability in China: A Stakeholder-Oriented Network Perspective. Sustainability, 10, 2090.

Shokoohi, R., \& Nikitas, A. (2017). Urban growth, and transportation in Kuala Lumpur: Can cycling be incorporated into Kuala Lumpur's transportation system?. Case Studies on Transport Policy, 5(4), 615-626.

Sun, F., Chen, P., \& Jiao, J. (2018). Promoting public bike-sharing: A lesson from the unsuccessful Pronto system. Transportation Research Part D: Transport and Environment, 63, 533-547.

van Waes, A., Farla, J., Frenken, K., de Jong , J., \& Raven, R. (2018 ). Business model innovation and socio-technical transitions. A new prospective framework with an application to bike sharing . Journal of Cleaner Production, 195 , 1300-1312 .

Vogel , P., Greiser, T., \& Mattfeld, D. (2011). Understanding Bike-Sharing Systems using Data Mining: Exploring Activity Patterns. Procedia - Social and Behavioral Sciences, 20, 514-523.

Wang, C., \& Zhang, P. (2012). The evolution of social commerce: The people, management, technology and information dimensions . Communications of the Association for Information Systems, 31(1), 105-127 .

Wang, K., Akar, G., \& Chen, Y. (2018). Bike sharing differences among millennials, Gen Xers and baby boomers: Lessons learnt from New York City's bike share . Transportation Research Part A: Policy and Practice, 116, 1-14 .

Wang, Y., Douglas, M. A., Hazen, B. T., \& Dresner, M. (2018). Be green and clearly be seen: How consumer values and attitudes affect adoption of bicycle sharing. Transportation Research Part F: Traffic Psychology and Behaviour, 58, 730-742.

Wang, M., \& Zhou, X. (2017). Bike-sharing systems and congestion: Evidence from US cities. Journal of Transport Geography, 65 , 147-154.

Winters, M., Hosford, K., \& Javaheri, S. (2019). Who are the 'super-users' of public bike share? An analysis of public bike share members in Vancouver, BC. Preventive Medicine Reports, 15, 100946.

Yang, Y., Heppenstall, A., Turner, A., \& Comber, A. (2019). A spatiotemporal and graphbased analysis of dockless bike sharing patterns to understand urban flows over the last mile. Computers, Environment and Urban Systems, 77, 101361.

Zhang, Z., Qian, C., \& Bian, Y. (2019). Bicycle-metro integration for the 'last mile': Visualizing cycling in Shanghai. Environment and Planning A: Economy and Space, 51(7), 1420-1423.

Zhang, L., Zhang, J., Duan, Z., \& Bryde, D. (2015). Sustainable bike-sharing systems: characteristics and commonalities across cases in urban China . Journal of Cleaner Production, 97 , 124-133. 